\title{
Global Analysis of WOX Transcription Factor Gene Family in Brassica napus Reveals Their Stress- and Hormone-Responsive Patterns
}

\author{
Mang-Mang Wang ${ }^{1,2}$, Ming-Ming Liu ${ }^{1,2}$, Feng Ran ${ }^{1}$, Peng-Cheng Guo ${ }^{1,2}$, Yun-Zhuo Ke ${ }^{1,2}$, \\ Yun-Wen Wu ${ }^{1}$, Jing Wen 1,2, Peng-Feng $\mathrm{Li}^{1,2}$, Jia-Na Li ${ }^{1,2}$ and Hai Du ${ }^{1,2, *}$ \\ 1 College of Agronomy and Biotechnology, Southwest University, Chongqing 400715, China; \\ mangmangwang16@126.com (M.-M.W.); mingmingliu95@163.com (M.-M.L.); fengran91@126.com (F.R.); \\ gpc930813@163.com (P.-C.G.); kyz2014@email.swu.edu.cn (Y.-Z.K.); wuyunwen97@163.com (Y.-W.W.); \\ luckywenjing93@126.com (J.W.); pengfengli17@126.com (P.-F.L.); ljn1950@swu.edu.cn (J.-N.L.) \\ 2 Academy of Agricultural Sciences, Southwest University, Chongqing 400715, China \\ * Correspondence: haidu81@126.com; Tel.: +86-182-2348-0008
}

Received: 28 September 2018; Accepted: 30 October 2018; Published: 5 November 2018

\begin{abstract}
The plant-specific WUSCHEL-related homeobox (WOX) transcription factor gene family is important for plant growth and development but little studied in oil crops. We identified and characterized 58 putative WOX genes in Brassica napus (BnWOXs), which were divided into three major clades and nine subclades based on the gene structure and conserved motifs. Collinearity analysis revealed that most BnWOXs were the products of allopolyploidization and segmental duplication events. Gene structure analysis indicated that introns/exons and protein motifs were conserved in each subclade and RNA sequencing revealed that BnWOXs had narrow expression profiles in major tissues and/or organs across different developmental stages. The expression pattern of each clade was highly conserved and similar to that of the sister and orthologous pairs from Brassica rapa and Brassica oleracea. Quantitative real-time polymerase chain reaction showed that members of the WOX4 subclade were induced in seedling roots by abiotic and hormone stresses, indicating their contribution to root development and abiotic stress responses. 463 proteins were predicted to interact with BnWOXs, including peptides regulating stem cell homeostasis in meristems. This study provides insights into the evolution and expression of the WOX gene family in B. napus and will be useful in future gene function research.
\end{abstract}

Keywords: WOX family; Brassica napus L.; phylogenetic analysis; expression analysis

\section{Introduction}

The WUSCHEL-related homeobox (WOX) family is a large group of transcription factors (TFs), specifically found in plants and characterized by the presence of 60-66 amino acids that constitute a DNA-binding domain, called a homeodomain (HB domain) [1]. The HB domain is highly conserved and important for the function of WOX protein family members, which are also characterized by the presence of a helix-loop-helix-turn-helix secondary structure.

Previous reports have shown that WOX genes play a wide variety of roles in plant development and growth processes such as embryonic patterning, stem cell maintenance and organ formation $[2,3]$. In Arabidopsis thaliana, there are at least 15 WOX genes (AtWOXs), classified into three clades: an ancient clade (WOX10, WOX13 and WOX14), intermediate clade (WOX8, WOX9, WOX11 and WOX12) and modern clade (WUS and WOX1-7) [2,4,5]. The functions of AtWOXs have been well studied and shown to be generally distinct in members of different clades and conserved in members of the same clade. For example, members of the modern clade maintain apical stem cells $[6,7]$ and members of 
the intermediate clade mainly regulate zygote development and early embryo morphogenesis $[8,9]$, while members of the ancient clade regulate root development [10]. The functions of WOXs in many other plants are also conserved and similar to those of their Arabidopsis homologs. For instance, in rice (Oryza sativa) and maize (Zea mays), the orthologs of AtWOX5 (OsWOX5 and ZmWOX5) play similar roles in the root meristem development [11,12]; in Norway spruce (Picea abies), PaWOX8 and PaWOX9 are orthologs of the members of the AtWOX8/9 subcladeand determine the cell fate during early embryo patterning [13]; and PtWUS in Populus trichocarpa corresponds to AtWUS, which is involved in shoot apical meristem (SAM) development.

Brassica napus L. (B. napus) has a high economic value because it is one of the main commercial sources of cooking oil. B. napus is an allotetraploid species (AnAnCnCn; $n=19$ ), which originated from the recent hybridization of two base diploid genomes of Brassica rapa L. (B. rapa; AnAn; $n=10$ ) and Brassica oleracea L. (B. oleracea; $\mathrm{CnCn} ; n=9$ ) [14]. Currently, there is a lack of detailed genomic information on the WOX gene family of $B$. napus, but it is of interest to explore the evolution and expression mechanisms of WOX genes (WOXs) between the Brassica An and Cn (BnAn and BnCn) sub-genomes [15]. Furthermore, understanding the structural relationships between Arabidopsis and $B$. napus would facilitate the prediction of B. napus WOX genes that remain uncharacterized.

In the present study, we performed a comprehensive genome-wide analysis of the WOX gene family in B. napus and identified 58 putative genes (BnWOXs) with open reading frames (ORFs). Phylogenetic analysis classified these BnWOXs into three clades, characterized by the integration of sequence features, chromosomal location, phylogenetic relationship, subcellular localization, interacting proteins, collinearity and expression patterns. In addition, quantitative real-time polymerase chain reaction (qRT-PCR) analysis showed that members of the WOX4 subclade were induced by abiotic stresses or hormones in seedling roots, suggesting their function in stress tolerance. Overall, our study will serve as a foundation for future research on the functional roles of WOXs in B. napus.

\section{Results}

\subsection{Identification of 58 BnWOX Genes and Their Physicochemical Properties}

To identify the WOX-encoding genes in the B. napus genome, a preliminary BLASTP search was performed using the HB domain sequences of known Arabidopsis WOX proteins as queries. In each case, a large number of deduced amino acid sequences ( $>50$ candidates) containing WOX or WOX-like repeats were obtained. Only hits with E-values of $<1.0$ were considered as members of the WOX gene family. The redundant candidate sequences were discarded from our data set, according to their chromosomal locations. We were then able to identify 58 typical, non-redundant WOX genes in the B. napus genome; those had complete ORF regions and encoded proteins with typical WOX features, which we verified using PROSITE (http:/ / www.expasy.org/tools/scanprosite/). To distinguish these genes, we provisionally named them BnWOX1 to BnWOX58 based on their order on the corresponding chromosomes (Table 1). We also identified 27 and 30 non-redundant WOX genes in B. rapa (BrWOXs) and B. oleracea (BoWOXs), respectively, using the same method (Table S1). Physicochemical property analysis showed that the corresponding BnWOX proteins (BnWOXs) varied in length from 121 to 390 amino acids; their molecular weight ranged from 13.96 to $71.82 \mathrm{kDa}$ and the isoelectric points were 5.08-9.58. Subcellular localization analysis demonstrated that all 58 proteins were located in the nucleus (Table 1). 
Table 1. Features of the 58 BnWOX genes from Brassica napus, identified in this study.

\begin{tabular}{|c|c|c|c|c|c|c|c|c|}
\hline Gene & Genome ID & Chromosome & $\begin{array}{l}\text { Protein Length } \\
\text { (Amino Acids) }\end{array}$ & $\begin{array}{l}\text { cDNA Length } \\
\text { (bp) }\end{array}$ & Introns/Exons & $\mathrm{pI}$ & $\begin{array}{c}\text { Molecular } \\
\text { Weight (Da) }\end{array}$ & $\begin{array}{l}\text { Subcellular } \\
\text { Localization }\end{array}$ \\
\hline BnWOX01 & BnaA01g01910D & chrA01 & 256 & 940 & $2 / 3$ & 5.72 & $28,458.4$ & Nucleus \\
\hline BnWOX02 & BnaA01g34040D & chrA01 & 289 & 870 & $3 / 4$ & 6.48 & $31,778.6$ & Nucleus \\
\hline BnWOX03 & BnaA02g07100D & chrA02 & 175 & 528 & $2 / 3$ & 6.36 & $19,236.2$ & Nucleus \\
\hline BnWOX04 & BnaA02g24210D & chrA02 & 320 & 1053 & $2 / 3$ & 6.96 & $35,591.2$ & Nucleus \\
\hline BnWOX05 & BnaA02g36550D & chrA02 & 302 & 909 & $2 / 3$ & 7.09 & $34,348.7$ & Nucleus \\
\hline BnWOX06 & BnaA03g22070D & chrA03 & 226 & 681 & $1 / 2$ & 9.33 & 26,087 & Nucleus \\
\hline BnWOX07 & BnaA03g53400D & chrA03 & 263 & 1036 & $2 / 3$ & 5.3 & $29,170.3$ & Nucleus \\
\hline BnWOX08 & BnaA04g16520D & chrA04 & 234 & 705 & $1 / 2$ & 9.32 & $27,143.2$ & Nucleus \\
\hline BnWOX09 & BnaA05g09770D & chrA05 & 390 & 1173 & $2 / 3$ & 8.35 & $43,341.9$ & Nucleus \\
\hline BnWOX10 & BnaA05g18600D & chrA05 & 251 & 756 & $2 / 3$ & 9.63 & $28,555.1$ & Nucleus \\
\hline BnWOX11 & BnaA05g22250D & chrA05 & 351 & 1287 & $3 / 4$ & 8.59 & $40,110.9$ & Nucleus \\
\hline BnWOX12 & BnaA05g27750D & chrA05 & 191 & 718 & $1 / 2$ & 8.87 & 22,361 & Nucleus \\
\hline BnWOX13 & BnaA05g32800D & chrA05 & 326 & 981 & $3 / 4$ & 6.63 & 35,661 & Nucleus \\
\hline BnWOX14 & BnaA06g14590D & chrA06 & 203 & 768 & $2 / 3$ & 5.43 & $22,850.2$ & Nucleus \\
\hline BnWOX15 & BnaA06g25450D & chrA06 & 294 & 885 & $2 / 3$ & 7.81 & $33,475.7$ & Nucleus \\
\hline BnWOX16 & BnaA07g02390D & chrA07 & 302 & 909 & $2 / 3$ & 6.85 & $34,305.6$ & Nucleus \\
\hline BnWOX17 & BnaA07g11310D & chrA07 & 133 & 402 & $1 / 2$ & 7.89 & $15,260.3$ & Nucleus \\
\hline BnWOX18 & BnaA08g04100D & chrA08 & 256 & 942 & $2 / 3$ & 9.31 & $29,143.6$ & Nucleus \\
\hline BnWOX19 & BnaA08g14960D & chrA08 & 239 & 720 & $3 / 4$ & 5.73 & $27,383.7$ & Nucleus \\
\hline BnWOX20 & BnaA09g09400D & chrA09 & 316 & 1022 & $3 / 4$ & 6.89 & 35,862 & Nucleus \\
\hline BnWOX21 & BnaA09g18650D & chrA09 & 267 & 804 & $4 / 5$ & 6.41 & $30,920.3$ & Nucleus \\
\hline BnWOX22 & BnaA09g45340D & chrA09 & 275 & 901 & $3 / 4$ & 6.28 & 31,962 & Nucleus \\
\hline BnWOX23 & BnaA10g12360D & chrA10 & 210 & 633 & $1 / 2$ & 6.83 & $23,085.3$ & Nucleus \\
\hline BnWOX24 & BnaA10g16970D & chrA10 & 274 & 949 & $2 / 3$ & 6.34 & $30,342.5$ & Nucleus \\
\hline BnWOX25 & BnaA10g24790D & chrA10 & 192 & 579 & $1 / 2$ & 9.17 & $22,114.9$ & Nucleus \\
\hline BnWOX26 & BnaAnng01480D & chrAnn_random & 191 & 576 & $1 / 2$ & 6.66 & $21,832.3$ & Nucleus \\
\hline BnWOX27 & BnaAnng11050D & chrAnn_random & 353 & 1062 & $3 / 4$ & 6.97 & $40,197.6$ & Nucleus \\
\hline BnWOX28 & BnaAnng35720D & chrAnn_random & 166 & 501 & $2 / 3$ & 8.99 & $19,173.4$ & Nucleus \\
\hline BnWOX29 & BnaAnng36010D & chrAnn_random & 121 & 366 & $1 / 2$ & 9.61 & $13,962.8$ & Nucleus \\
\hline BnWOX30 & BnaAnng41310D & chrAnn_random & 247 & 847 & $1 / 2$ & 8.77 & $27,574.8$ & Nucleus \\
\hline BnWOX31 & BnaC01g03050D & $\operatorname{chrC} 01$ & 265 & 1016 & $2 / 3$ & 5.67 & $29,250.4$ & Nucleus \\
\hline BnWOX32 & BnaC01g40590D & chrC01 & 289 & 972 & $3 / 4$ & 6.48 & $31,778.6$ & Nucleus \\
\hline BnWOX33 & BnaC02g02200D & chrC02 & 191 & 576 & $1 / 2$ & 5.18 & $46,938.7$ & Nucleus \\
\hline BnWOX34 & BnaC02g07490D & chrC02 & 283 & 852 & $2 / 3$ & 5.08 & $71,825.3$ & Nucleus \\
\hline BnWOX35 & BnaC02g08530D & chrC02 & 121 & 366 & $1 / 2$ & 6.09 & $31,334.6$ & Nucleus \\
\hline
\end{tabular}


Table 1. Cont

\begin{tabular}{|c|c|c|c|c|c|c|c|c|}
\hline Gene & Genome ID & Chromosome & $\begin{array}{l}\text { Protein Length } \\
\text { (Amino Acids) }\end{array}$ & $\begin{array}{l}\text { cDNA Length } \\
\text { (bp) }\end{array}$ & Introns/Exons & pI & $\begin{array}{c}\text { Molecular } \\
\text { Weight (Da) }\end{array}$ & $\begin{array}{l}\text { Subcellular } \\
\text { Localization }\end{array}$ \\
\hline BnWOX36 & BnaC02g10040D & chrC02 & 199 & 600 & $1 / 2$ & 5.95 & $21,671.7$ & Nucleus \\
\hline BnWOX37 & BnaC02g32050D & $\mathrm{chrC02}$ & 320 & 1277 & $2 / 3$ & 6.96 & $35,542.2$ & Nucleus \\
\hline BnWOX38 & BnaC03g18850D & $\mathrm{chrC03}$ & 397 & 1303 & $3 / 4$ & 6.57 & $44,157.5$ & Nucleus \\
\hline BnWOX39 & BnaC03g26450D & $\mathrm{chrC03}$ & 222 & 669 & $1 / 2$ & 9.26 & $25,657.6$ & Nucleus \\
\hline BnWOX40 & BnaC03g40380D & chrC03 & 350 & 1053 & $3 / 4$ & 7.72 & $40,024.6$ & Nucleus \\
\hline BnWOX41 & BnaC03g62360D & chrC03 & 171 & 641 & $1 / 2$ & 7.65 & $19,697.3$ & Nucleus \\
\hline BnWOX42 & BnaC04g39860D & chrC04 & 236 & 711 & $1 / 2$ & 9.3 & $27,260.4$ & Nucleus \\
\hline BnWOX43 & BnaC04g46990D & $\mathrm{chrC04}$ & 195 & 588 & $2 / 3$ & 5.51 & $21,785.3$ & Nucleus \\
\hline BnWOX44 & BnaC05g25380D & chrC05 & 251 & 908 & $2 / 3$ & 9.58 & 28,526 & Nucleus \\
\hline BnWOX45 & BnaC05g41930D & chrC05 & 191 & 718 & $1 / 2$ & 8.87 & $22,485.1$ & Nucleus \\
\hline BnWOX46 & BnaC05g48100D & chrC05 & 339 & 1020 & $3 / 4$ & 5.98 & $37,085.8$ & Nucleus \\
\hline BnWOX47 & BnaC07g06960D & chrC07 & 302 & 2162 & $2 / 3$ & 7.36 & $34,407.9$ & Nucleus \\
\hline BnWOX 48 & BnaC07g15200D & chrC07 & 195 & 588 & $2 / 3$ & 5.35 & $21,686.1$ & Nucleus \\
\hline BnWOX 49 & BnaC07g45680D & chrC07 & 261 & 1022 & $2 / 3$ & 5.23 & $29,076.2$ & Nucleus \\
\hline BnWOX50 & BnaC08g04810D & chrC08 & 255 & 938 & $2 / 3$ & 9.43 & $28,912.4$ & Nucleus \\
\hline BnWOX51 & BnaC08g39150D & chrC08 & 290 & 873 & $4 / 5$ & 5.93 & $33,988.5$ & Nucleus \\
\hline BnWOX52 & BnaC09g34650D & chrC09 & 210 & 633 & $1 / 2$ & 6.58 & $23,144.4$ & Nucleus \\
\hline BnWOX53 & BnaC09g40100D & chrC09 & 276 & 1794 & $2 / 3$ & 5.97 & $30,547.8$ & Nucleus \\
\hline BnWOX54 & BnaC09g49730D & chrC09 & 192 & 579 & $1 / 2$ & 8.99 & $22,099.8$ & Nucleus \\
\hline BnWOX55 & BnaCnng49040D & chrCnn_random & 209 & 579 & $2 / 3$ & 5.48 & $23,619.1$ & Nucleus \\
\hline BnWOX56 & BnaCnng49570D & chrCnn_random & 280 & 843 & $1 / 2$ & 5.97 & $31,047.2$ & Nucleus \\
\hline BnWOX57 & BnaCnng51820D & chrCnn_random & 351 & 1314 & $3 / 4$ & 8.4 & $40,001.6$ & Nucleus \\
\hline BnWOX58 & BnaCnng62530D & chrCnn_random & 390 & 1954 & $2 / 3$ & 8.35 & $43,232.7$ & Nucleus \\
\hline
\end{tabular}




\subsection{Phylogenetic Analysis of the BnWOX Gene Family}

To determine the evolutionary relationships of the BnWOX gene family with those of Arabidopsis and the $B$. napus ancestor species, we constructed neighbor-joining (NJ) and maximum-likelihood (ML) phylogenetic trees of 130 WOX proteins, from B. napus (58), B. rapa (27), B. oleracea (30) and Arabidopsis (15), based on the multi-alignment of their HB domains using MEGA 7.0 [16] and PhyML 3.0 [17], respectively.

Our results showed that the NJ and ML tree topologies were highly congruent (Figure 1 and Figure S1). In the phylogenetic trees, the 130 WOX members clustered into three main clades: modern, intermediate and ancient clades (Figure 1). The number of WOX genes in the modern clade (72 genes) was greater than that in the ancient ( 24 genes) and intermediate ( 34 genes) clades, indicating the gene expansion in higher plants. Our data were consistent with those of previous reports, which indicated that the WOX gene family was chronologically divided into three clades (i.e., ancient, intermediate and modern/WUS clades) [4]. In each clade, the numbers of genes from these four species were usually different and each Arabidopsis WOX gene (AtWOX) generally had more than one ortholog. Thus, the modern clade consisted of 33 BnWOXs, 15 BoWOXs, 16 BrWOXs and 8 AtWOXs; the intermediate clade included 14 BnWOXs, 9 BoWOXs, 7 BrWOXs and 4 AtWOXs; and the ancient clade had 11 BnWOXs, 6 BoWOXs, 7 BrWOXs and 3 AtWOXs. This distribution showed that the number of genes constantly increased with the evolution of the gene family.

We further divided the candidate WOX genes into nine subclades: WUS, WOX1, WOX2, WOX3, WOX4, WOX5/7, WOX6, WOX11/12 and WOX8/9, based on the bootstrap values and the topology of the phylogenetic tree. There were seven subclades in the modern clade and two in the intermediate clade (Figure 1). In the ancient clade, only homologs of WOX13 were found in non-Brassicaceae species [3,18-20]; however, we found that homologs of WOX10/14 existed in all of the four Brassicaceae, which indicates that WOX10/14 may be unique to Brassicaceae [21]. In addition, the WOX1/WOX6 homologs were divided into two subclades (WOX1 and WOX6), with high bootstrap values.

\subsection{Sequence Analysis of B. napus WOX Domains}

To compare the sequence features, we performed a multiple alignment analysis of the HB domains of the 58 BnWOXs using MAFFT with the default parameters [22]. The sequence logos and the secondary structures of the HB domains were generated on the Weblogo (http: / / weblogo.berkeley. edu/logo.cgi) and PRABI (https:/ / npsa-prabi.ibcp.fr/cgi-bin/npsa_automat.pl?page=npsa_sopma. html) platforms (Figure 2). Our results showed that the HB domains were highly conserved and commonly contained helix-loop-helix-turn-helix structures, which were either 63 or 64 amino acid residues in length, with the exception of BnWOX23, BnWOX52, BnWOX13 and BnWOX36, which had a short amino acid deletion at the C-terminus due to incomplete genome information (Figure 2). Consistent with previous reports [3], there was a conserved $Y$ (Tyr) residue insertion after the 17th amino acid in the HB domains of all AtWUS homologs, resulting in a total of 63 amino acids (Figure 2). In addition, 16 amino acid residues were completely conserved and mainly located in the third helix, which may play an important role in gene function $[1,3]$. Interestingly, there were also some conserved subclade-specific substitutions (e.g., at 8 th, 37th, 20th and 23rd amino acid position) in the HB domains in the modern clade (Figure 2). However, the functional properties of these sites need to be further predicted and/or confirmed by mutational analysis, which was beyond the scope of the current study.

The FYWFQNH, FYWFQNR and YNWFQNR motifs (from 50th to 56th amino acid in the HB domain) have been reported as representative markers for the three main clades, respectively $[18,23]$. In the present study, we confirmed these motifs in B. napus as well but the $Y$ residue in the YNWFQNR motif was replaced by C (Cys) in BnWOX19 and BnWOX41 in the ancient clade. Together, our analysis of the protein sequences indicated that the HB domains are highly conserved in BnWOXs. 


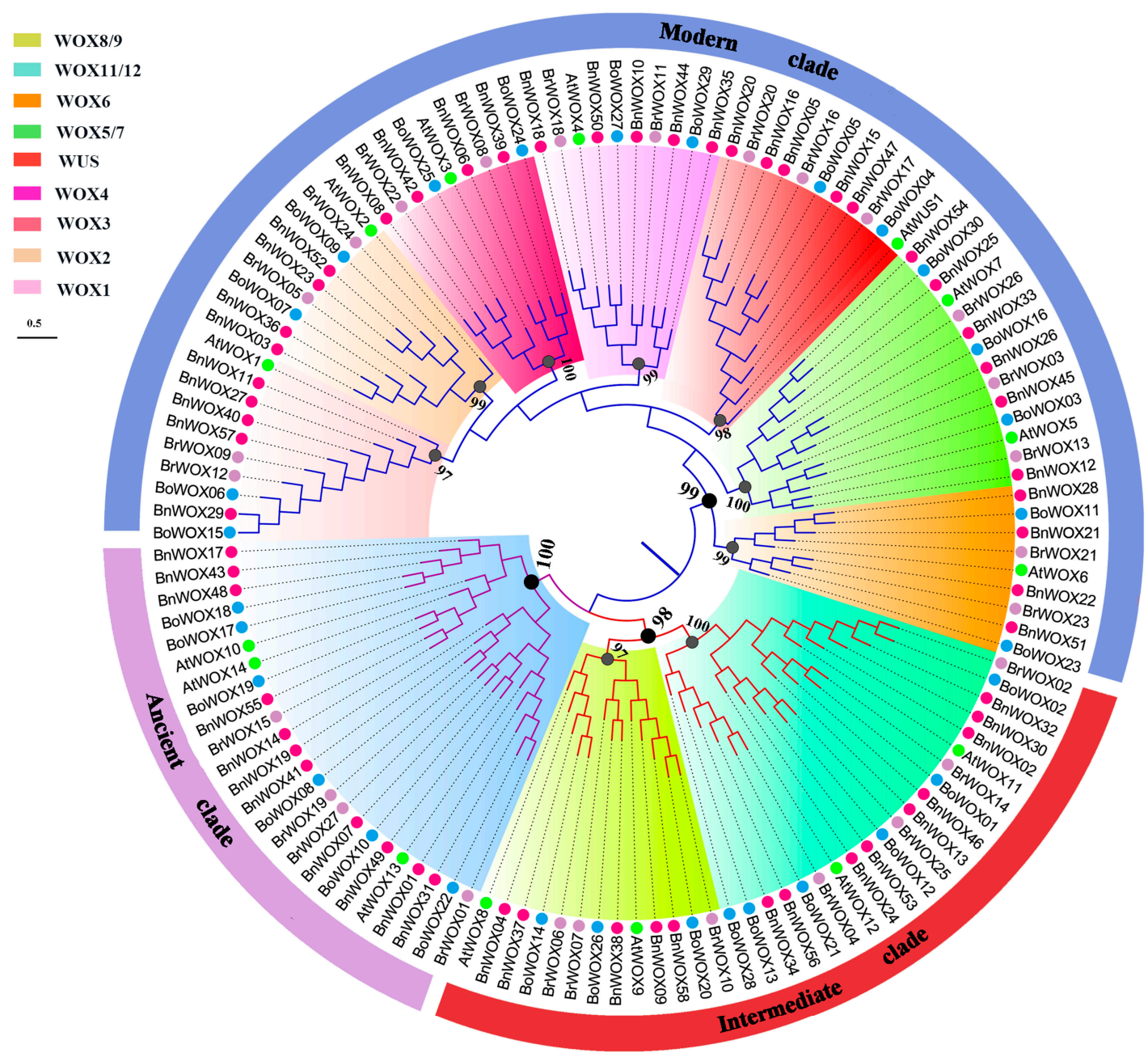

Figure 1. Phylogenetic relationships of 130 WOX genes. The phylogenetic tree was generated from the alignment of 130 WOX protein homeodomain sequences, with 1000 bootstrap replicates. The WOX genes from Arabidopsis (At: 15), B. rapa (Br: 27), B. oleracea (Bo: 30) and B. napus (Bn: 58) are shown as green, purple, blue and red dots, respectively. The outer circle represents three clades, marked in purple, blue and brown colors. Nine subclades were identified and are marked in different background colors; bootstrap values are shown near the nodes. 


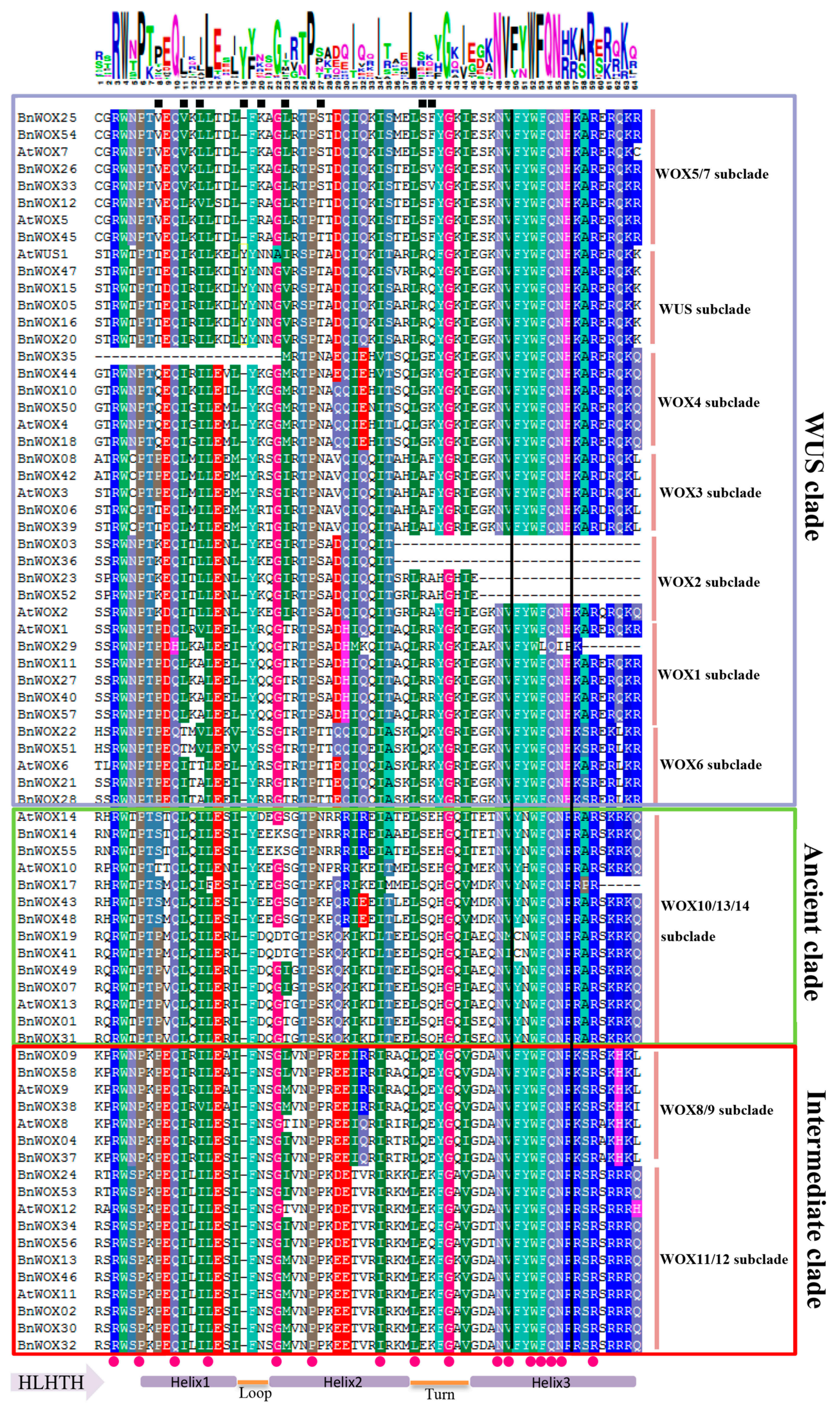

Figure 2. Homeodomain sequence analysis in WOX proteins from B. napus and Arabidopsis. The sequence logos are based on the alignments and the WOX family members contain the typical helix-loop-helix-turn-helix structure. Completely conserved residues are represented by red dots and WUS subclade-specific residues are indicated by black squares. Black boxes indicate marker sequences for the clades and green, red and purple boxes show the ancient, intermediate and WUS clades, respectively, with subclades. The conserved $Y$ residue insertion at the 18 th position is also indicated. 


\subsection{Gene Structure and Intron Pattern Analysis}

Structural diversity of genes can provide an important clue for the function and evolution of multigene families [24]; therefore, in the present study, we analyzed the gene structure of WOXs in $B$. napus, B. rapa, B. oleracea and Arabidopsis (Figure S2).

We did not observe intron insertions in the HB domains of the 73 WOXs from Arabidopsis and $B$. napus. Members of the same subclade generally had the same or similar exon/intron patterns, except for the WOX6 and WOX11/12 subclades, which showed complex exon-intron patterns and significant variation in the number of introns present (Figure 3). The HB domains were located in the first exon in the WOX5 subclade and in the second exon in the WOX10/14 subclade, although they were commonly conserved in members of the same clade or subclade (Figure 3). Moreover, 12 conserved intron patterns were found in the BnWOX gene family (Table S2). Members of the ancient clade had two intron insertion sites; the first was conserved throughout all WOX members but the second differed between WOX13 and WOX10/14 homologs. In the intermediate clade, we identified three intron patterns and the intron insertion sites were similar in the WOX8/9 subclade, while members of the WOX11/12 subclade showed different intron insertion patterns and the number of introns was variable. Interestingly, the sequences at the C-terminal end of the genes in the WOX11/12 and WOX8/9 subclades were highly homologous; accordingly, several genes across these two subclades had a conserved intron insertion site in this region (Figure 3). Similarly, in the modern clade, the intron insertion sites were generally highly conserved in each subclade, except the WOX6 subclade, which had several members with an additional intron insertion site at the $\mathrm{C}$ - or $\mathrm{N}$-terminus. Moreover, we found that very few intron patterns were different between members of the WOX1/WOX6 and WOX5/WUS subclades because of their high homology. These results indicated that these clades or subclades may have had a common ancestor and that intron insertion might be a driving force of functional differentiation during evolution.

We predicted that 25 BnWOXs were complementary to 31 microRNA sequences (Table S3), which suggests that the WOX gene family may be regulated by microRNAs. Moreover, we found that $32 \%$, $12 \%$ and $56 \%$ of BnWOXs belonged to the ancient, intermediate and modern clades, respectively, thus showing the concentration of the genes in the modern clade. This result indicated that the members of the clade might participate in a larger number of biological processes, as will be discussed below; however, the mismatch rate was as high as $33 \%$ so that the accuracy of these findings needs further experimental verification.

Overall, our results suggested that the intron loss/gain had occurred in WOXs outside the HB domain during the evolution of the gene family in Brassicaceae and the highly conserved or similar intron patterns that we identified further support the data of our phylogenetic analysis and classification.

\subsection{Conserved Motif Analysis of B. napus WOX Proteins}

Specific motifs are generally related to functional conversion and diversion and conserved motifs could be identified in the 58 BnWOX proteins using the MEME software and sequence alignment. 


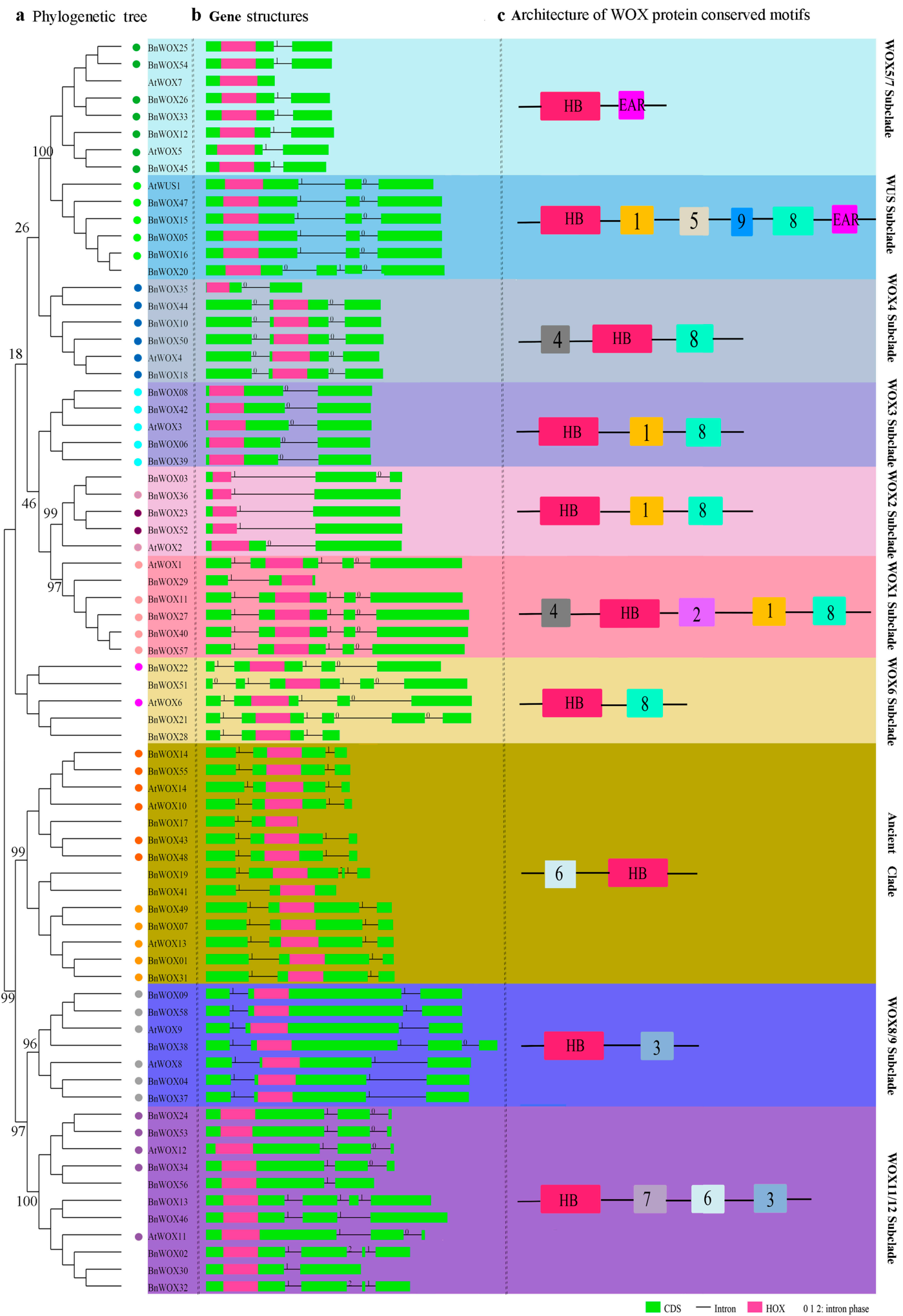

Figure 3. Gene structure and conserved motifs in WOXs from Arabidopsis and B. napus. (a) The neighbor-joining tree was generated from the alignment of 73 WOX genes from Arabidopsis and B. napus. The different background colors represent the nine subclades, which are supported by high bootstrap values. The colored dots indicate the 12 conserved intron insertion patterns. (b) Gene structures were generated using the Gene Structure Display Server (GSDS 2.0) [25]. Green boxes indicate exons; black lines indicate introns and pink boxes represent the HB domains. Numbers 0,1 and 2 represent intron phases. (c) Conserved motifs were detected using MEME and are shown as boxes of different colors. Motif sequences are provided in Table S4. 
Eleven conserved motifs (motifs 1-11) were identified in the full-length BnWOX proteins (Table S4). With the exception of the conserved HB domain (motif 10) present in the members of all clades, the remaining domains (motifs) were only distributed in members of a given clade or subclade. In most cases, the same clade and subclade shared similar motif compositions, which further supported our phylogenetic analysis and classification data based on the homology of the HB domains in the WOX protein family. This indicated that some motifs were only shared by WOX proteins within the same clade or subclade, thus being subclade specific. For example, motifs 8 and 3 were present in all members of the modern and intermediate clades, respectively, while motif 7 was specific to the WOX11/12 subclade, which may be related to the acquisition of a novel function. However, several motifs were shared among clades or subclades, suggesting a common origin. For instance, motif 6 was common for the ancient clade and WOX11/12 subclade, which may indicate their close evolutionary relationship. No ancient clade-specific motif was found in this study.

In Arabidopsis, there are three functional domains in members of several subclades of the modern clade and these domains contribute significantly to the protein functions $[3,26]$. Thus, the acidic region (motif 5) can potentially function as an activator domain [2]; the WUS box (TLXLFP; motif 8) is essential for the transcriptional repressor activity and involved in the regulation of leaf blade outgrowth [5]; and the EAR-like motif (motif 11) is involved in transcriptional repression [27]. In the present study, although the acidic region and EAR-like (LXLXL) motif were not identified in the BnWOX proteins by MEME, we confirmed these two motifs in the modern clade by multi-sequence alignment analysis. The WUS box existed in most members of the modern clade, except those in the WOX7 subclade, while the acidic region was only present in the WUS subclade proteins and the EAR-like motif was merely present in the members of the WUS and WOX5/7 subclades, except AtWOX7 (Figure 3). However, the acidic region was not strictly conserved among the WUS members from B. napus and similar results were obtained in Solanaceae species [28], where several sites were substituted in the motif. Future research should examine whether these amino acid substitutions impact the functions of candidate genes in B. napus. Taken together, the organization of conserved motifs in each clade or subclade of the BnWOX gene family encoded proteins was strongly conserved. To some extent, these specific motifs may contribute to the functional divergence and conservation of BnWOX proteins.

\subsection{Chromosomal Distribution and Duplication of B. napus WOX Genes}

To investigate the relationships between genetic divergence and gene duplications within the $B$. napus WOX gene family, we determined the chromosomal location of the candidate BnWOXs based on the genome annotation information from the Genoscope database and visualized the data with $\mathrm{R}$ [29].

Our results showed that all but nine genes were located in the unanchored random regions and the last 49 BnWOXs were unevenly distributed on 18 of the 19 chromosomes (except for ChrC06) (Figure 4). For example, there were five genes on $\mathrm{ChrA05}$ and $\mathrm{ChrC02}$ each and only one on ChrA04. In general, BnWOXs were randomly arranged on the top and/or bottom of the chromosomes. B. napus (AnAnCnCn; $n=19)$ is an allotetraploid derived from the recent hybridization between B. rapa (AnAn; $n=10)$ and B. oleracea ( $\mathrm{CnCn} ; n=9)$ [14] and is, therefore, an ideal species for studying the effects of polyploidy on WOX gene expansion. In the present study, we also identified 27 and 30 WOX genes in the $B$. rapa and $B$. oleracea genomes, respectively and found that these genes were similar to those in the An and Cn sub-genomes of B. napus [14].

Collinearity analysis revealed that there were strong orthologs among the B. napus, B. rapa, $B$. oleracea and Arabidopsis WOX genes (Figure 4). There was a tripling in Brassica species after diversion from their common ancestor with Arabidopsis [14]. Thus, one Arabidopsis WOX should theoretically correspond to three orthologs in B. rapa and B. oleracea. However, the synteny between WOX genes of B. rapa and B. oleracea and their Arabidopsis homologs was less than expected (13:27:25; Table S6), indicating that duplicated genes might have been lost during evolution [30]. As expected, 42 of the 58 BnWOXs (70\%) found in the B. napus genome had a syntenic relationship, among which 32 BnWOXs were inherited from BoWOXs (18 genes) and BrWOXs (14 genes). These results indicate 
that allotetraploidy was the main force for the rapid expansion of the WOX gene family in B. napus. Moreover, eight (19\%) BnWOXs were obtained by segmental duplication events, including two, three and three genes in the ancient, modern and intermediate clades, respectively (Table S5), while one gene was obtained by a homologous exchange. In addition, we found that eight (53\%) AtWOXs, seven (23\%) BoWOXs and $17(60 \%)$ BrWOXs resulted from segmental duplication and whole-genome duplication in Arabidopsis, B. oleracea and B. rapa, respectively. Similar results have been reported for cotton [20]. We found only one putative tandem duplication, located on ChrA06 (BnaA06g25460D and BnaA06g25450D) of B. napus, which indicates that tandem duplications may have contributed less to the expansion of the WOX gene family. Overall, our results indicate that the expansion of the WOX gene family in the B. napus genome was mainly due to whole-genome duplication (polyploidy) and segmental duplication.

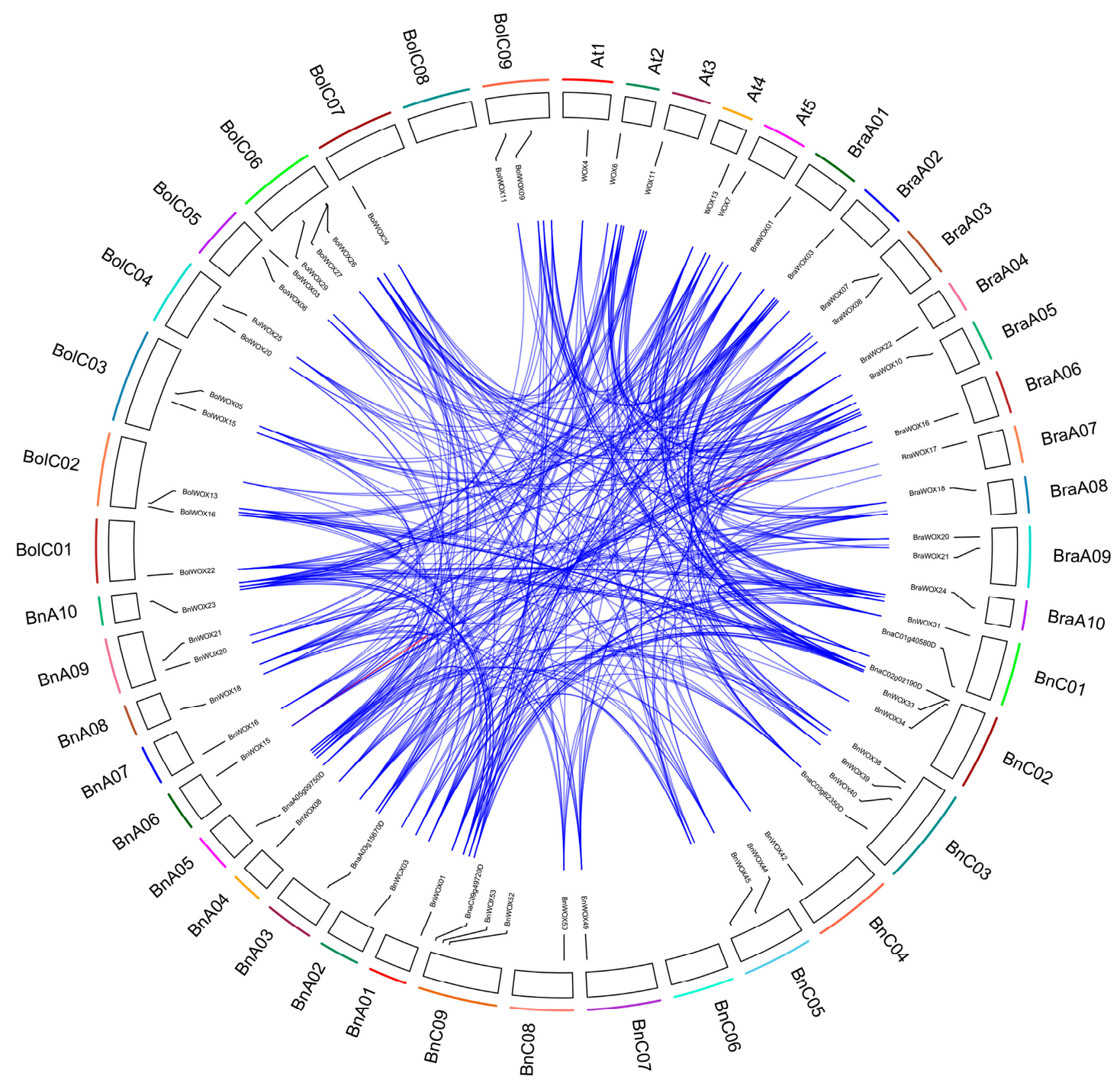

Figure 4. Collinearity analysis of WOX genes from Arabidopsis, B. rapa, B. oleracea and B. napus. The outer circle indicates the chromosome numbers and the inner circle indicates the location of AtWOXs, BnWOXs, BrWOXs and BoWOXs on the chromosomes. The blue lines link two syntenic WOX genes from B. napus, Arabidopsis, B. rapa and B. oleracea. The red line indicates a tandem duplication.

\subsection{Prediction of BnWOX Protein Interaction Networks in B. napus}

It has been reported that some WOX proteins function by forming complexes with other proteins [10]. However, there is still no genome-wide overview of the protein interaction network 
of the WOX family members. Hence, on the basis of the data publicly available in STRING [31], we predicted and constructed a protein-protein interaction network for BnWOXs, based on their orthology with AtWOXs and visualized these findings with Cytoscape [32].

First, we predicted 38 interacting protein pairs for 10 AtWOXs, based on the Arabidopsis data in STRING (interaction score $>0.65$ ). Among the 10 AtWOXs, one was in the intermediate clade, two in the ancient clade and seven in the modern clade, suggesting an increasing protein interaction trend in the modern clade. Among the 38 predicted proteins, $27 \%$ were peptides, $30 \%$ were TFs and the rest were other types of proteins. It is, therefore, clear that most proteins that interact with WOXs are peptides, such as CLAVATA3 (CLV3)/ESR-RELATED 40 (CLE40) and CLV3 (Table S7). CLE peptides are a well-known group of post-translationally modified signal molecules involved in cell division in SAM, the root apical meristem (RAM) and vascular meristem, which can respond to stress signals $[10,33,34]$. Previous reports have demonstrated that AtWUS, AtWOX4, AtWOX5 and AtWOX14 can regulate cell division in SAM, vascular meristem and RAM [10,35], respectively, while WUS-CLV interactions were shown to establish a feedback loop between stem cells and the underlying regulatory center [7]. In addition, the results of the present study showed that AtWOXs could interact with some TFs, such as GRAS, SCR and SHR (Table S7). It is interesting that homologs of WOX5, SCR and SHR have been reported to be important for root development [36]. Although their interaction was not demonstrated, the data indicated that WOX proteins might regulate the root development by interacting with GRAS proteins.

Based on the orthologous relationship between B. napus and Arabidopsis, a total of 463 interacting protein pairs were predicted for BnWOXs (Figure 5). Our results showed that protein interactions might be common for this gene family and that WOX proteins, especially those in the modern clade, might play a conserved role in regulating proliferation and differentiation of meristem stem cells. Our study provides important information needed to further investigate the molecular mechanisms of BnWOXs in Brassica species.

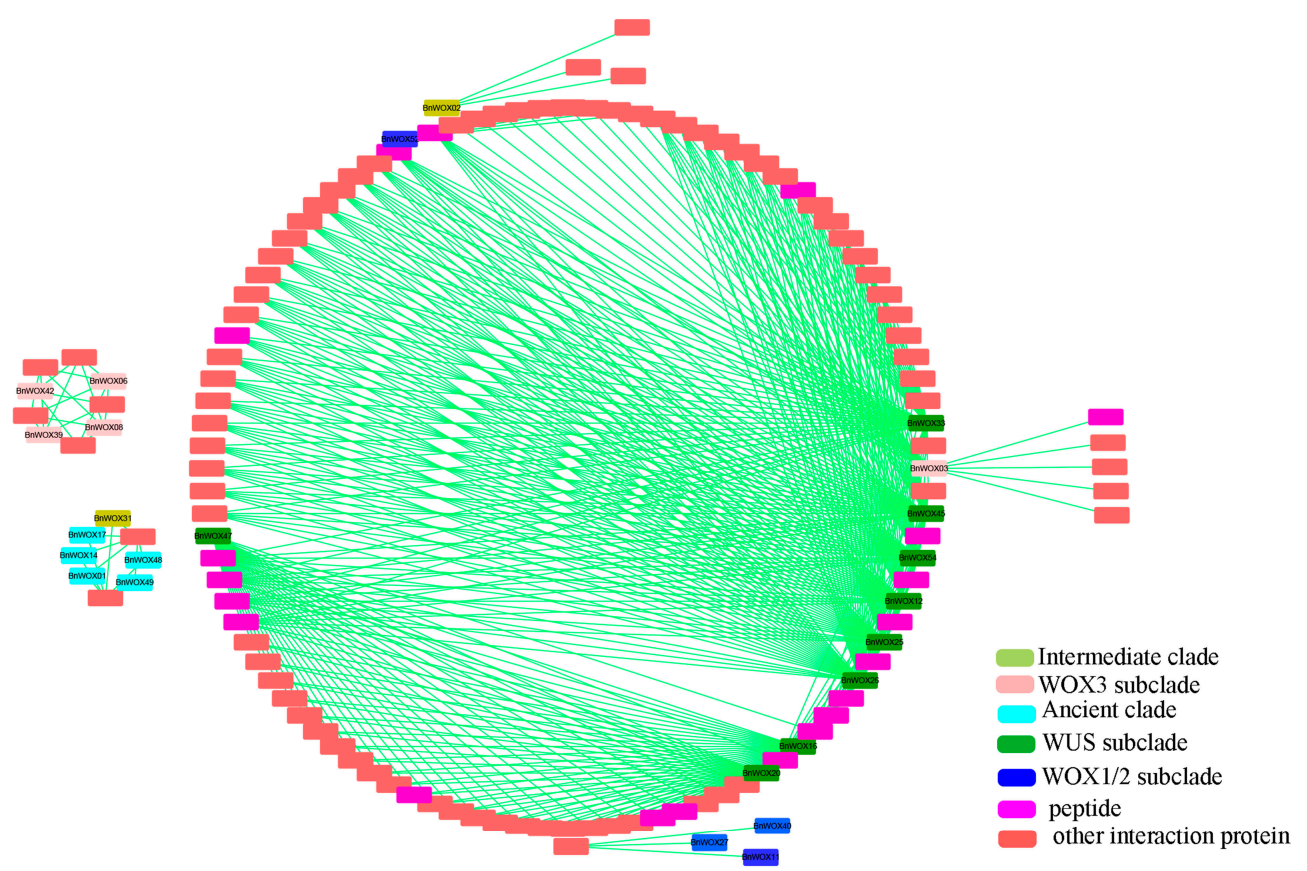

Figure 5. Protein interaction network of BnWOXs in B. napus. Different color blocks show BnWOXs from different clades and subclades, as well as different types of interacting proteins.

\subsection{Expression Analysis of WOX Genes at Different Developmental Stages in B. napus}

Gene expression is associated with biological function of the encoded protein; therefore, we examined the expression patterns of the 58 BnWOXs in 50 B. napus tissues/organs, based on the 
RNA-seq data from the Gene Expression Omnibus database at the National Center of Biotechnology Information. To make the image used for the expression analysis more intuitive, we excluded from the heatmap (Figure 6) data on 18 BnWOXs with no or weak expression (fragments per kilobase of transcript per million mapped reads $(\mathrm{FPKM})<1$ ), which are most likely pseudogenes or expressed under certain conditions. We found that all BnWOXs were likely to be expressed in a limited number of vegetative tissues and reproductive organs, with relatively more genes expressed in root, stem and seed tissues, suggesting possible temporal and spatial expression patterns. Moreover, expression patterns of members of each clade were relatively conserved. We did not observe any expression of the genes from the ancient clade, except for BnWOX19, BnWOX48, BnWOX41, BnWOX43 and BnWOX13; the remaining genes showed conserved expression patterns in roots, stems and pistils at flowering stages (Figure 6). Fourteen members of the intermediate clade were conserved and preferentially expressed in seeds, especially in the seed coat and embryo tissues, suggesting that they may contribute to seed maturation and embryonic development. This result was consistent with those of a previous report, which indicated that members of the WOX8/9 subclade regulated tissue proliferation during embryonic development in Arabidopsis [37]. In the modern clade, BnWOXs showed a relatively wider expression pattern than did those from the other two clades and were highly expressed in the roots, stems, flowers and seeds at different stages, which indicated that members of the modern clade may play tissue-specific roles. In general, the expression patterns were different for members of different subclades in the modern clade but very similar within the same subclade. For example, BnWOX10, BnWOX18, BnWOX44 and BnWOX50 from the WOX4 subclade were highly expressed in roots and stems, while BnWOX27, BnWOX40 and BnWOX11 from the WOX1 subclade showed high levels of expression in the pistil. The relatively wider expression patterns of members of the modern clade were consistent with the data from previous reports, which showed that this clade originated later and underwent clear functional divergence from the other clades in this gene family [2]. Together, these results show that BnWOXs generally have narrow expression profiles, being predominantly expressed in roots, stems and seeds and that the expression patterns in each clade or subclade reflect functional conservation.

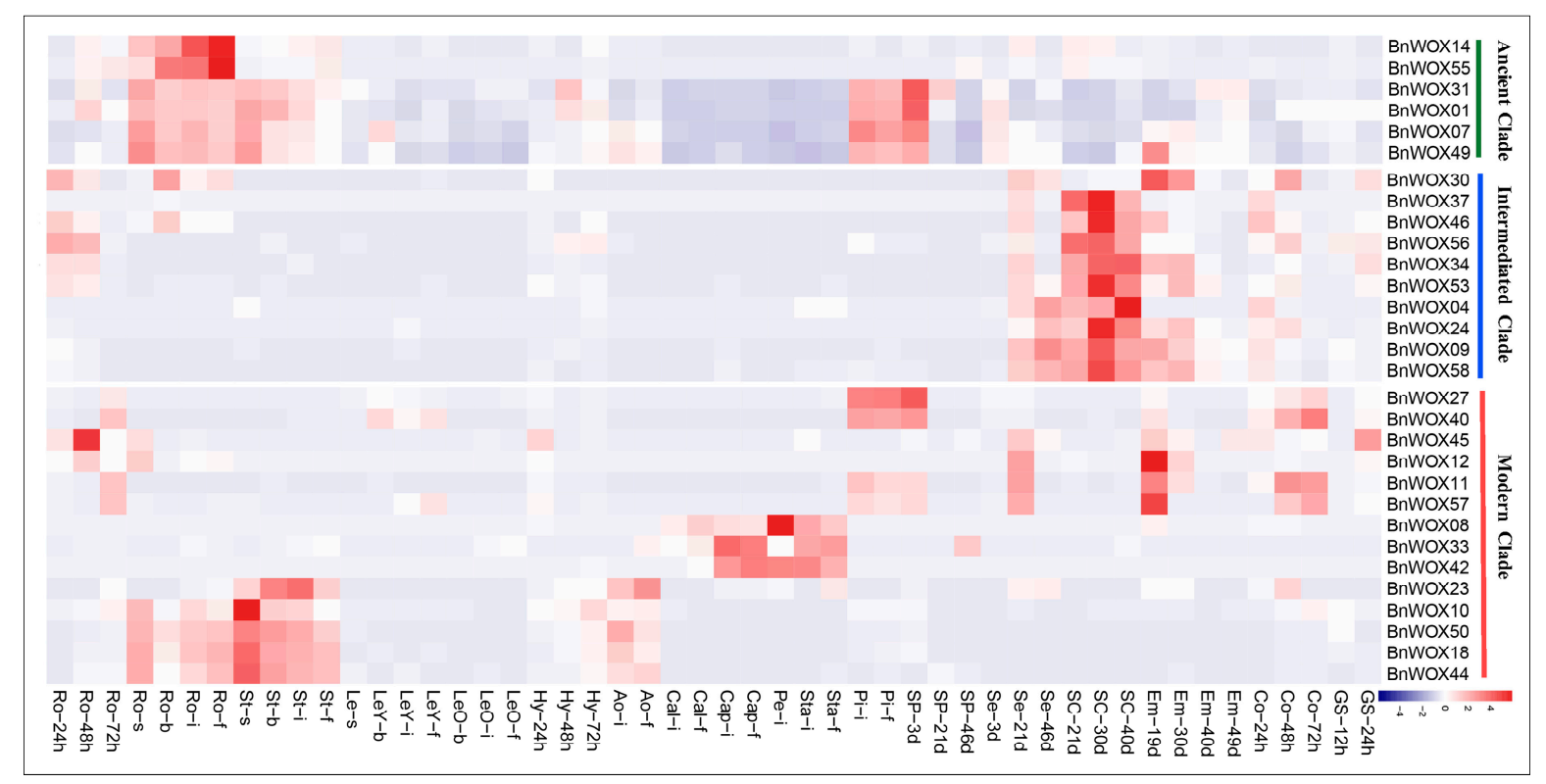

Figure 6. Patterns of BnWOX gene expression in different tissues during plant development. In the color bar at the lower right side of the figure, blue represents little or no expression, and red represents a high level of expression. Ro = root, $\mathrm{St}=$ stem, $\mathrm{Le}=$ leaf, $\mathrm{Sp}=$ silique pericarp, $\mathrm{Sc}=$ seed coat, $\mathrm{Em}=$ embryo, $\mathrm{Ao}=$ anthocaulus, $\mathrm{Se}=$ seed, $\mathrm{Hy}=$ hypocotyl, GS = germinating seed, Cap = capillament, $\mathrm{Pi}=$ pistil, $\mathrm{Cal}=$ calyx, $\mathrm{Co}=$ cotyledon and $\mathrm{Pe}=$ petal; $\mathrm{h}, \mathrm{d}, \mathrm{s}, \mathrm{b}, \mathrm{i}$ and $\mathrm{f}$ indicate hour, day, seeding, budding, initial flowering and full-bloom stages, respectively. BnWOXs with no or weak expression $($ FPKM $<1)$ were removed and the remaining family members were clustered by clades. 


\subsection{Responses of BnWOX Genes to Environmental Stresses and Phytohormone Induction}

Previous studies on WOX genes from various species have mainly focused on plant development $[38,39]$, while their responses to environmental stresses and hormone induction were seldom evaluated. Therefore, in the present study, a comprehensive expression analysis of BnWOXs, based on RNA-seq data, was performed in roots after treatment with five hormones, including the auxin indole-3-acetic acid (IAA), abscisic acid (ABA), the cytokinin 6-benzyladenine (6-BA), the ethylene precursor 1-aminocyclopropane-1-carboxylic acid (ACC) and gibberellic acid (GA). Our results showed no obvious changes in the expression levels of more than half of BnWOXs after phytohormone treatment; however, several members of the WOX4 subclade were induced in seedling roots, which indicated their possible roles in hormone responses (Figure S3).

To further elucidate these expression results and gain an insight into the expression of BnWOXs in response to abiotic stresses (salt and drought), four members of the WOX4 subclade (BnWOX10, BnWOX50, BnWOX44 and BnWOX18) were selected to investigate their responses to hormone, salt and polyethylene glycol (PEG) stresses using qRT-PCR to evaluate changes in gene expression. The results of qRT-PCR analysis were similar to those of our RNA-seq analysis of phytohormone treatment responses, that is, the members of the WOX4 subclade were differentially regulated by different stress treatments (Figure 7). Moreover, as a sister pair, BnWOX50 and BnWOX18 showed similar stress responses, being repressed by almost all exogenous phytohormones, although 6-BA, which downregulated the expression of these genes at 1, 3 and $6 \mathrm{~h}$, gradually reversed this inhibitory effect at 12 and $24 \mathrm{~h}$. Moreover, three-fold inhibition of BnWOX50 and BnWOX18 expression was observed after NaCl and PEG treatments, compared with their expression in the untreated control, indicating that these genes may be involved in drought and salt resistance. On the other hand, the BnWOX10 and BnWOX44 genes showed differential expression patterns under some stress conditions, while being similarly upregulated by ABA treatment and downregulated after $6 \mathrm{~h}$ of IAA treatment. The expression of BnWOX44 was upregulated by ACC, GA, 6-BA, NaCl and PEG treatments, while BnWOX10 was repressed by the same treatments, indicating functional divergence between these genes. Overall, members of the WOX4 subclade may play vital roles in responses to salt and drought stresses, as well as to phytohormones, which makes these genes candidates for further study of B. napus abiotic stress responses.

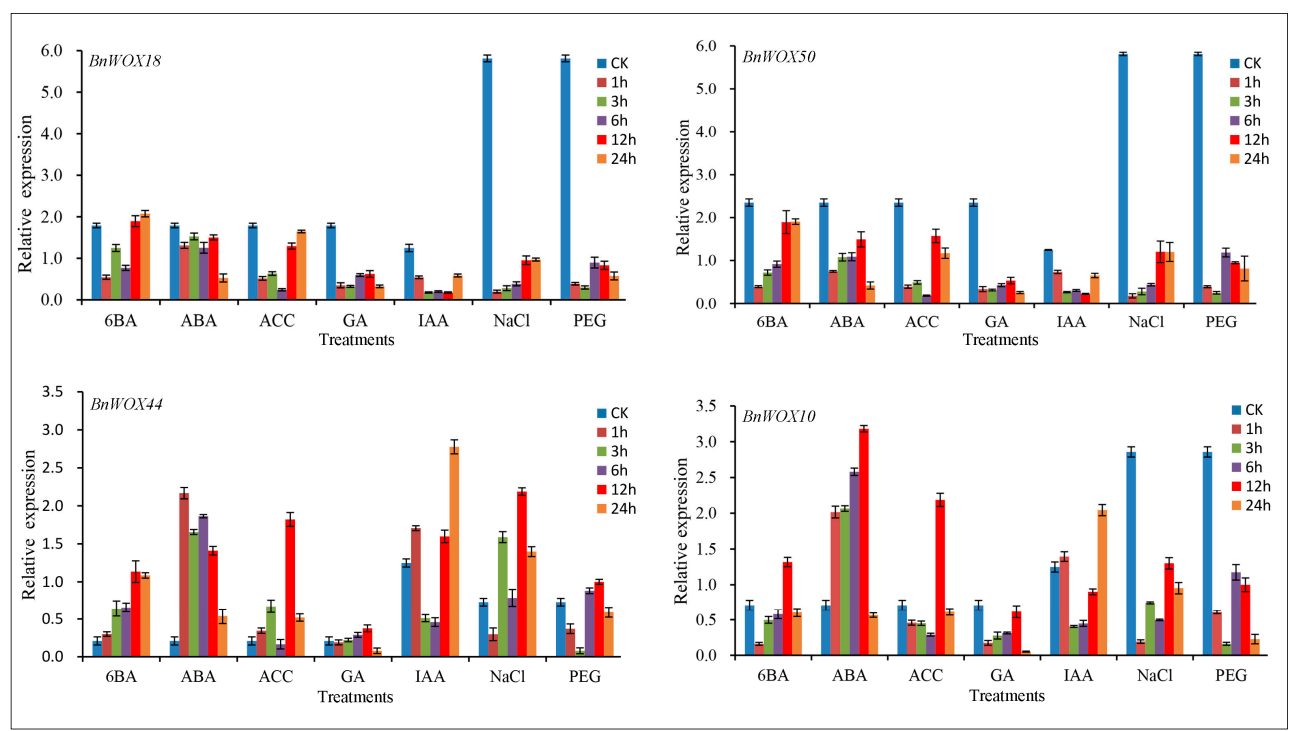

Figure 7. Expression levels of four BnWOXs after different stress treatments. Transcript levels were determined in seedling root samples by qRT-PCR under different stress treatment conditions. PEG simulated the drought stress and $\mathrm{NaCl}$ was used for salt stress. Gibberellic acid (GA), abscisic acid (ABA), indole-3-acetic acid (IAA), 1-aminocyclopropane-1-carboxylic acid (ACC) and 6-benzyladenine (6-BA) were used for phytohormone treatments. $\mathrm{CK}=$ control. Data are the mean \pm standard deviation of three independent experiments. 


\section{Discussion}

\subsection{Structural and Functional Conservation of the Plant WOX Gene Family}

The WOX gene family is specific to plants and has been identified in rice, sorghum, maize, Arabidopsis and Norway spruce $[3,12,13]$. WOX genes are well known to play key roles in the development and functional conservation in various plant species [2,6,8,40]. To confirm this conservation of the WOX gene family across land plants, we summarized the functions of WOX genes that have been characterized in plants to date (Table S11). We found that members of the ancient clade were mainly involved in the regulation of root development; members of the intermediate clade were involved in embryogenesis and morphological development and members of the modern clade were mainly involved in meristem maintenance. Although functional differentiation has occurred in the modern clade, the functions of members of each subclade are relatively conserved. For example, members of the WOX4 subclade in Arabidopsis and rice mainly promote vascularization [10]; the expression profile in B. napus in the present study was consistent with previous findings, further supporting functional conservation across different species.

Notably, we found that functional conservation of each clade or subclade was supported by a highly conserved gene structure, illustrated by conserved motif and intron patterns. The HB domain contains a helix-loop-helix-turn-helix structure [22], which can recognize sequence-specific targets in a precise spatial and temporal manner. Moreover, the domain is conserved in different species, thus maintaining its functional integrity [2,3]. In addition, we found representative markers, YNWFQNR, FYWFQNH and FYWFQNR, in all three clades in B. napus, while members of the modern and intermediate clades contained conserved substitutions at the $H$ and $R$ residues, which may be responsible for their functional diversification (Figure 3). Interestingly, we found that these representative markers and some conserved amino acid residues were located in the helix 3 region of the HB domain. Thus, we speculate that helix 3 plays a pivotal role in the functional differentiation between different clades. Although the functions of the proteins in the modern clade were relatively varied, they were conserved within the same subclade. There were also several conserved substitutions outside the helix 3 region in the modern clade; thus, 27\%,50\%, 19\% and 28\% of subclade-specific sites existed in the helix 1, loop, helix 2 and turn regions, respectively, indicating that the loop and turn regions were also main regions related to the functional differentiation in the modern clade (Figure 3).

We observed that the conserved motifs outside the HB domain were specific for each clade or subclade, indicating that these motifs were likely required for specific protein functions. In the modern clade, nearly all the members (except AtWOX7) shared a conserved WUS box. It has also been reported that STF/WOX1 interacted with TOPLESS (TPL)/TPL-related proteins through the conserved WUS box to regulate the blade outgrowth by mediating cell proliferation in Medicago truncatula [41]. Similarly, the results of the present study revealed that TPL could interact with WOX5 homologs, suggesting that this may be a common mechanism for the repressive function of the WUS box in members of the modern clade. The EAR-like domain was shared by members of the WUS and WOX5/7 subclades and has been reported to act as a transcriptional repressor [3]. In addition, a previous report has indicated that the acidic region was related to the activation of transcription of WOX proteins [2] and was only found in the WUS subclade. These results demonstrated that specific motifs were conserved in proteins belonging to certain clades and subclades and could play important roles in the functional conservation in the WOX gene family. Consequently, the other motifs identified in this study may also be critical for gene function, although their roles still need further experimental clarification.

Introns can be specifically inserted and conserved in plant genomes and are related to functional diversity as exons are lost or gained during evolution [18]. In the present study, we found that intron insertion sites were similar or conserved in the same clades or subclades (Table S2), which further supports the division and functional conservation of subclades. Members of the WOX4 subclade, which are involved in the maintenance of vegetative and reproductive meristems [10], shared conserved patterns of introns. Differences in the intron patterns between 
the WOX2 and WOX1 subclades corresponded to their involvement in zygotic apical cell and lateral organ development, respectively. Thus, the intron insertion patterns may contribute to functional conservation and differentiation.

The results of our analysis of the relationships of conserved motifs and intron patterns with the gene structure and expression profiles of BnWOXs indicate that there are homologous genes in each clade and/or subclade, which contribute to functional conservation in different plant species. The results presented here will be useful for selecting appropriate candidate genes for further functional research in B. napus.

\subsection{Conservation of Expression Profiles in Different Plants Supports Their Functional Conservation}

The expression pattern of a gene often correlates with its function and previous studies have investigated the expression of WOXs in rice and Arabidopsis [3,14,42]. To further confirm the functional conservation and differentiation during the evolution of land plants, we analyzed WOX gene expression patterns in rice, soybean, B. napus, B. oleracea and B. rapa (Figure S4). Our results indicated that the expression patterns of members of the intermediate and ancient clades were generally conserved and they were expressed at high levels in green pods and roots in soybean (Figure S5). However, the expression of ancient-clade genes differed between rice and soybean, indicating that functional differentiation occurred during the evolution of monocot and dicot plants.

As mentioned above, B. napus is a typical allotetraploid, making it an ideal model for studying the effect of naturally occurring polyploidy on genetic conservation [14]. To investigate the functional conservation and differentiation in the WOX gene family, we compared the expression patterns and sequence identities/similarities of the HB domains, as well as the full-length protein, gene and promoter sequences of 32 orthologous pairs between B. napus, B. oleracea and B. rapa and of 23 sister pairs from B. napus (Table S9). We found that BoWOXs and BrWOXs were also mainly expressed in vegetative (root, stem and leaf) and reproductive (flower and silique) organs. Most members of the intermediate clade showed no or very low expression levels compared to those of members of the other two clades, which may indicate that the former are pseudogenes or need to be induced by specific stresses. The expression of WOXs from the ancient and intermediate clades was conserved across B. napus, B. oleracea and B. rapa and was mainly observed in roots (Figure S3). Our results also showed that most sister pairs shared a very high degree of sequence identity, with $96.9 \%$ identity in the HB domains and $86.32 \%$ identity in the full-length proteins. Accordingly, $73 \%$ (17 of 23 ) of the BnWOX paralogs shared similar expression patterns. Among these sister pairs, $80 \%, 85 \%$ and $55 \%$ belonged to the ancient, intermediate and modern clades, respectively, implying that these genes are functionally redundant. For example, the BnWOX09 and BnWOX58 pair from the intermediate clade was highly expressed in seed tissues, while the BnWOX55 and BnWOX14 pair from the ancient clade was highly expressed in roots. In contrast, several sister pairs underwent neo-functionalization or sub-functionalization, especially in the modern clade, so that their expression patterns were slightly or obviously different. For instance, in the BnWOX24 and BnWOX53 pair, only BnWOX53 was highly expressed in the root (Figure 6). Furthermore, the promoter regions of sister pairs were significantly homologous, with the average sequence identity ranging from $20 \%$ to $86 \%$ (Table S9). In addition, the expression patterns of sister pairs were closely related to their promoter sequence identities. Thus, we found that several sister pair genes that were differentially expressed had high sequence identity in the $\mathrm{HB}$ regions but less identity in the promoter regions, suggesting that the functional divergence of homologous WOX genes may have first occurred in the promoter regions during their evolution.

In this study, we identified 18 and 14 orthologous pairs from the WOX gene family in B. rapa and B. oleracea using the identity in the HB domain, which was $93.6 \%$ and $89.9 \%$, respectively. However, the expression patterns of $62 \%$ of the orthologous pairs were slightly divergent or significantly different. For example, BnWOX53 and BoWOX12 were both expressed in roots but BnWOX53 was also expressed in seeds, indicating that functional divergence occurred during the evolution of these two genes. Interestingly, approximately $60 \%, 20 \%$ and $20 \%$ of the WOX genes that exhibited differential 
expression belonged to the modern, intermediate and ancient clades, respectively (Table S8), further supporting the hypothesis that the modern clade emerged recently in land plants and underwent functional diversification during the evolution. Furthermore, the average levels of identity of the promoter regions of orthologous pairs ranged from $8 \%$ to $99.7 \%$ and were generally much lower than those of the ORF regions, indicating that the promoter regions play vital roles in the functional diversity of WOX genes. Taken together, comparison of the expression patterns of WOX genes across different land plants revealed the major functional conservation and/or slight differentiation during the evolution of this gene family and indicated that the functional differentiation of these genes may be related to sequence diversity in their promoter regions.

\section{Materials and Methods}

\subsection{Identification of WOX Proteins and Phylogenetic Analysis of the B. napus Genome}

The sequences of 15 Arabidopsis WOX genes were downloaded from the TAIR Arabidopsis genome (http://www.arabidopsis.org/) [43] to aid in the identification of candidate genes encoding WOX proteins in B. napus. We performed a BLASTP search of the Genoscope genome database (http:/ /www. genoscope.cns.fr/brassicanapus/) using the DNA-binding domain protein sequences of the Arabidopsis WOX genes as queries. To verify the reliability of our results, the protein functional and structural domains were predicted by PROSITE profiling (http://www.expasy.org/tools/scanprosite/) [44] to confirm that each protein had the HB domain. We acquired the B. oleracea and B. rapa WOX protein sequences from Phytozome (https://phytozome.jgi.doe.gov/pz/portal.html) and BRAD (http:/ /brassicadb.org/brad/index.php) using the same method as that used for Arabidopsis.

The biochemical properties of the candidates were predicted using ExPASy [45] and subcellular localization was investigated using Cell-PLoc [46].

To gain insights into the evolutionary history of the WOX gene family in B. napus, B. rapa, $B$. oleracea and Arabidopsis, we constructed an NJ tree based on a multiple sequence alignment of the $\mathrm{HB}$ domains, using MEGA version 7.0 [16] with the following parameters: Poisson correction, pairwise deletion and a bootstrap with 1000 replicates. Tree files were viewed and edited with FigTree v1.3.1 (http:/ / tree.bio.ed.ac.uk/software/figtree/). The MEGA 7.0 program was used to estimate the best model for the multiple sequence alignment of the HB domains by default. The JJT amino acid substitution model with estimation of the gamma distribution shape parameter (JJT $+\mathrm{G}+\mathrm{I}$ ) was suggested to be the best evolutionary models, based on the Akaike information criterion (AIC)statistics. An ML tree was constructed using PhyML 3.0 [17] with 100 replicates and the JJT + G + I model.

\subsection{Sequence, Gene Structure and Conserved Motif Analysis and Construction of the Protein Interaction Network}

To analyze the sequence features of the BnWOXs proteins, we performed a multiple alignment analysis of the HB domains using MAFFT version 7 with the default parameters (https:/ / mafft.cbrc. $\mathrm{jp}$ /alignment/server/). To obtain optimized alignments, the deduced amino acid sequences were adjusted manually in MEGA 7.0 and BioEdit 7.0 (http:/ / www.psc.edu/biomed/genedoc/) [16] with the default parameters. The intron patterns, including the distribution, position and phases and the HB domain positions in the B. napus WOX genes were analyzed using the GSDS software 2.0 (http://gsds.cbi.pku.edu.cn/) [25]. The intron insertion information for the WOX genes of Arabidopsis, B. rapa and B. oleracea was acquired from Phytozome (https:/ / phytozome.jgi.doe.gov/pz/portal.html).

The MEME version 4.11.1 [47] program was used to identify potential protein motifs, in addition to the HB domain, using the following parameter settings for the distribution of motifs: the maximum number of motifs, 10; the minimum width of a motif, 6 ; and the maximum width of a motif, 50. Only motifs with an E-value $\leq 1 \times 10^{-10}$ were used for further analysis. The interaction network was constructed based on the orthologs of BnWOXs in Arabidopsis using the STRING platform 
(https://string-db.org/?tdsourcetag=s_pctim_aiomsg) and visualized with Cytoscape version 3.4.0 (Chongqing, China).

\subsection{Chromosomal Location and Synteny Analysis}

The gene loci for B. napus WOX genes were extracted from the Genoscope genome database. The synteny relationships of WOX genes from Arabidopsis, B. napus, B. oleracea and B. rapa were acquired from CoGe (https:/ / genomevolution.org/coge/). The R package [29] was used to view the chromosomal locations of the candidates and their collinearity.

\subsection{Analysis of Expression Profiles of BnWOXs at Different Developmental Stages and After Hormone and Stress Treatment}

The temporal and spatial expression patterns of candidate BnWOXs were further analyzed using the RNA-seq data from 50 different tissues, which included roots, stems, leaves, flowers, seeds and siliques from the B. napus cultivar "Zhongshuang 11" (ZS11) at different developmental stages (e.g., germination, seedling, budding, initial flowering and full-bloom stages). Seedling roots treated with hormones (e.g., IAA, GA, 6-BA, ABA and ACC) were also collected for analysis. The data were analyzed with Cluster 3.0 (Chongqing, China) [32] and a heatmap was drawn using the R package.

For qRT-PCR, seeds of ZS11 were obtained from the College of Agriculture and Biotechnology, Southwest University (Chongqing, China) and germinated on Petri dishes. At the five-leaf stage, seedlings were treated with Hoagland's liquid medium, which contained a 15\% $(w / v)$ PEG 6000 solution to simulate a drought condition, $200 \mathrm{mM} \mathrm{NaCl}$, or phytohormones $(50 \mu \mathrm{M} \mathrm{ABA}, 120 \mu \mathrm{M} \mathrm{GA}$, $75 \mu \mathrm{M}$ 6-BA, $60 \mu \mathrm{M}$ ACC and $10 \mu \mathrm{M}$ IAA), and grown in an artificial climate chamber at $25^{\circ} \mathrm{C}$ under a 14-h/10-h (day/night) photoperiod. Root tissue was harvested at 0, 1, 3, 6, 12 and $24 \mathrm{~h}$ of treatment, immediately frozen in liquid nitrogen and stored at $-80^{\circ} \mathrm{C}$ for RNA isolation.

Extraction of total RNA from root samples and subsequent cDNA synthesis were performed as described previously [48]. The SYBR Premix ExTaq ${ }^{\mathrm{TM}}$ II kit (Takara Biotechnology, Dalian, China) was used for qRT-PCR amplification in a CFX Connect ${ }^{\mathrm{TM}}$ real-time PCR system (Bio-Rad, Chongqing, China) and the SYBR Green PrimeScript RT-PCR kit (Takara Biotechnology). Each reaction was conducted in a $10 \mu \mathrm{L}$ volume and contained $5 \mu \mathrm{L}$ of PCR master mix, $2.5 \mu \mathrm{L}$ of double distilled $\mathrm{H}_{2} \mathrm{O}\left(\mathrm{ddH}_{2} \mathrm{O}\right), 2 \mu \mathrm{L}$ of diluted template and $0.25 \mu \mathrm{L}$ of each gene-specific primer (Table S10). Three biological replicates were performed and three technical replicates were taken for each biological replicate. The Actin7 gene (GenBank accession no. AF024716) was used as an internal control. The reaction conditions for real-time PCR were as follows: initial denaturation at $95{ }^{\circ} \mathrm{C}$ for $3 \mathrm{~min}$, followed by 40 cycles of denaturation at $95{ }^{\circ} \mathrm{C}$ for $10 \mathrm{~s}$ and annealing at $58^{\circ} \mathrm{C}$ for $30 \mathrm{~s}$. The relative gene expression levels were calculated using the $2^{-\Delta \Delta C t}$ method [49].

Supplementary Materials: Supplementary materials can be found at http:/ /www.mdpi.com/1422-0067/19/11/ $3470 /$ s1.

Author Contributions: H.D. developed the conception of the study; M.-M.W., J.-N.L. and H.D drafted and revised the manuscript. M.-M.W., H.D., M.-M.L., J.W., F.R., Y.-W.W. and P.-C.G. contributed to data analysis. M.-M.W., H.D., Y.-Z.K. and P.-F.L. conceived and designed the experiments. All authors reviewed and approved the final manuscript.

Funding: This work was supported by the National Natural Science Foundation of China (31471528 and 31671727), the "111" Project of China (B12006) and the National Basic Research Program of China (973 Program; 2015CB150201).

Conflicts of Interest: The authors declare no conflict of interest. 


\section{Abbreviations}

$\begin{array}{ll}\text { ABA } & \text { abscisic acid } \\ \text { ACC } & \text { 1-aminocyclopropane-1-carboxylic acid } \\ \text { CLV3 } & \text { CLAVATA3 } \\ \text { CLE40 } & \text { CLAVATA3/ESR-RELATED 40 } \\ \text { FPKM } & \text { fragments per kilobase of transcript per million mapped reads } \\ \text { GA } & \text { gibberellic acid } \\ \text { GSDS } & \text { gene structure display server } \\ \text { HB domain } & \text { homeodomain } \\ \text { IAA } & \text { indole-3-acetic acid } \\ \text { ORF } & \text { open reading frame } \\ \text { PEG } & \text { polyethylene glycol } \\ \text { qRT-PCR } & \text { quantitative real-time polymerase chain reaction } \\ \text { RAM } & \text { root apical meristem } \\ \text { RNA-seq } & \text { RNA sequencing } \\ \text { SAM } & \text { shoot apical meristem } \\ \text { TPL } & \text { TOPLESS } \\ \text { WOX } & \text { WUSCHEL-related homeobox } \\ \text { ZS11 } & \text { "Zhongshuang 11" }\end{array}$

\section{References}

1. Mayer, K.F.; Schoof, H.; Haecker, A.; Lenhard, M.; Jürgens, G.; Laux, T. Role of WUSCHEL in regulating stem cell fate in the Arabidopsis shoot meristem. Cell 1998, 95, 805. [CrossRef]

2. Vandenbussche, M.; Horstman, A.; Zethof, J.; Koes, R.; Rijpkema, A.S.; Gerats, T. Differential recruitment of WOX transcription factors for lateral development and organ fusion in petunia and Arabidopsis. Plant Cell 2009, 21, 2269-2283. [CrossRef] [PubMed]

3. Zhang, X.; Zong, J.; Liu, J.; Yin, J.; Zhang, D. Genome-wide analysis of WOX gene family in rice, sorghum, maize, Arabidopsis and poplar. J. Integr. Plant Biol. 2010, 52, 1016-1026. [CrossRef] [PubMed]

4. Haecker, A.; Gross-Hardt, R.; Geiges, B.; Sarkar, A.; Breuninger, H.; Herrmann, M.; Laux, T. Expression dynamics of WOX genes mark cell fate decisions during early embryonic patterning in Arabidopsis thaliana. Development 2004, 131, 657-668. [CrossRef] [PubMed]

5. Lin, H.; Niu, L.; McHale, N.A.; Ohme-Takagi, M.; Mysore, K.S.; Tadege, M. Evolutionarily conserved repressive activity of WOX proteins mediates leaf blade outgrowth and floral organ development in plants. Plant Signal. Behav. 2013, 110, 366-371. [CrossRef] [PubMed]

6. Sarkar, A.K.; Luijten, M.; Miyashima, S.; Lenhard, M.; Hashimoto, T.; Nakajima, K.; Scheres, B.; Heidstra, R.; Laux, T.; Sarkar, A.K.; et al. Conserved factors regulate signalling in Arabidopsis thaliana shoot and root stem cell organizers. Nature 2007, 446, 811-814. [CrossRef] [PubMed]

7. Schoof, H.; Lenhard, M.; Haecker, A.; Mayer, K.F.; Jürgens, G.; Laux, T. The stem cell population of Arabidopsis shoot meristems in maintained by a regulatory loop between the CLAVATA and WUSCHEL genes. Cell 2000, 100, 635-644. [CrossRef]

8. Breuninger, H.; Rikirsch, E.; Hermann, M.; Ueda, M.; Laux, T. Differential expression of WOX genes mediates apical-basal axis formation in the Arabidopsis embryo. Dev. Cell 2008, 14, 867-876. [CrossRef] [PubMed]

9. Wu, X.; Dabi, T.; Weigel, D. Requirement of homeobox gene STIMPY/WOX9 for Arabidopsis meristem growth and maintenance. Curr. Biol. 2005, 15, 436-440. [CrossRef] [PubMed]

10. Etchells, J.P.; Provost, C.M.; Mishra, L.; Turner, S.R. WOX4 and WOX14 act downstream of the PXY receptor kinase to regulate plant vascular proliferation independently of any role in vascular organisation. Development 2013, 140, 2224-2234. [CrossRef] [PubMed]

11. Kamiya, N.; Nagasaki, H.; Morikami, A.; Sato, Y.; Matsuoka, M. Isolation and characterization of a rice WUSCHEL-type homeobox gene that is specifically expressed in the central cells of a quiescent center in the root apical meristem. Plant J. 2003, 35, 429-441. [CrossRef] [PubMed] 
12. Nardmann, J.; Werr, W. The shoot stem cell niche in angiosperms: Expression patterns of WUS orthologues in rice and maize imply major modifications in the course of mono- and dicot evolution. Mol. Biol. Evol. 2006, 23, 2492-2504. [CrossRef] [PubMed]

13. Zhu, T.; Moschou, P.N.; Alvarez, J.M.; Sohlberg, J.J.; Arnold, S.V. WUSCHEL-RELATED HOMEOBOX 8/9 is important for proper embryo patterning in the gymnosperm Norway spruce. J. Exp. Bot. 2014, 65, 6543-6552. [CrossRef] [PubMed]

14. Chalhoub, B.; Denoeud, F.; Liu, S.; Parkin, I.A.; Tang, H.; Wang, X.; Chiquet, J.; Belcram, H.; Tong, C.; Samans, B. Early allopolyploid evolution in the post-Neolithic Brassica napus oilseed genome. Science 2014, 345, 950-953. [CrossRef] [PubMed]

15. He, Z.; Cheng, F.; Li, Y.; Wang, X.; Parkin, I.A.; Chalhoub, B.; Liu, S.; Bancroft, I. Construction of Brassica A and $C$ genome-based ordered pan-transcriptomes for use in rapeseed genomic research. Data Brief 2015, 4, 357-362. [CrossRef] [PubMed]

16. Kumar, S.; Stecher, G.; Tamura, K. MEGA7: Molecular evolutionary genetics analysis version 7.0 for bigger datasets. Mol. Biol. Evol. 2016, 33, 1870. [CrossRef] [PubMed]

17. Stéphane, G.; Frédéric, D.; Jean-François, D.; Olivier, G. Estimating maximum likelihood phylogenies with PhyML. Methods Mol. Biol. 2009, 537, 113-137. [CrossRef]

18. Cao, Y.; Han, Y.; Meng, D.; Li, G.; Li, D.; Abdullah, M.; Jin, Q.; Lin, Y.; Cai, Y. Genome-wide analysis suggests the relaxed purifying selection affect the evolution of WOX genes in Pyrus bretschneideri, Prunus persica, Prunus mume, and Fragaria vesca. Front. Genet. 2017, 8, 78. [CrossRef] [PubMed]

19. Nardmann, J.; Werr, W. Symplesiomorphies in the WUSCHEL clade suggest that the last common ancestor of seed plants contained at least four independent stem cell niches. New Phytol. 2013, 199, 1081-1092. [CrossRef] [PubMed]

20. Yang, Z.; Gong, Q.; Qin, W.; Yang, Z.; Cheng, Y.; Lu, L.; Ge, X.; Zhang, C.; Wu, Z.; Li, F. Genome-wide analysis of WOX genes in upland cotton and their expression pattern under different stresses. BMC Plant Biol. 2017, 17, 113. [CrossRef] [PubMed]

21. Lian, G.; Ding, Z.; Wang, Q.; Zhang, D.; Xu, J. Origins and Evolution of WUSCHEL-related homeobox protein family in plant kingdom. Sci. World J. 2014, 2014, 534140. [CrossRef] [PubMed]

22. Katoh, K.; Standley, D.M. MAFFT multiple sequence alignment software version 7: Improvements in performance and usability. Mol. Biol. Evol. 2013, 30, 772-780. [CrossRef] [PubMed]

23. Zeng, M.; Hu, B.; Li, J.; Zhang, G.; Ying, R.; Huang, H.; Wang, H.; Xu, L. Stem cell lineage in body layer specialization and vascular patterning of rice root and leaf. Sci. Bull. 2016, 61, 847-858. [CrossRef]

24. Hedman, H.; Zhu, T.; Arnold, S.V.; Sohlberg, J.J. Analysis of the WUSCHEL-RELATED HOMEOBOX gene family in the conifer picea abies reveals extensive conservation as well as dynamic patterns. BMC Plant Biol. 2013, 13, 89. [CrossRef] [PubMed]

25. Hu, B.; Jin, J.; Guo, A.Y.; Zhang, H.; Luo, J.; Gao, G. GSDS 2.0: An upgraded gene feature visualization server. Bioinformatics 2014, 31, 1296. [CrossRef] [PubMed]

26. Ikeda, M.; Ohme-Takagi, M. Arabidopsis WUSCHEL is a bifunctional transcription factor that acts as a repressor in stem cell regulation and as an activator in floral patterning. Plant Cell 2009, 21, 3493-3505. [CrossRef] [PubMed]

27. Paponov, I.A.; Teale, W.; Lang, D.; Paponov, M.; Reski, R.; Rensing, S.A.; Palme, K. The evolution of nuclear auxin signalling. BMC Evol. Biol. 2009, 9, 126. [CrossRef] [PubMed]

28. Li, X.; Hamyat, M.; Liu, C.; Salman, A.; Gao, X.; Guo, C.; Wang, Y.; Guo, Y. Identification and characterization of the WOX family genes in five Solanaceae Species reveal their conserved roles in peptide signaling. Genes 2018, 9, 260. [CrossRef] [PubMed]

29. Zhang, H.; Meltzer, P.; Davis, S. RCircos: An R package for Circos 2D track plots. BMC Bioinform. 2013, 14, 244. [CrossRef] [PubMed]

30. Lynch, M.; Conery, J.S. The evolutionary fate and consequences of duplicate genes. Science 2000, 290, 1151-1155. [CrossRef] [PubMed]

31. Szklarczyk, D.; Morris, J.H.; Cook, H.; Kuhn, M.; Wyder, S.; Simonovic, M.; Santos, A.; Doncheva, N.T.; Roth, A.; Bork, P. The STRING database in 2017: Quality-controlled protein-protein association networks, made broadly accessible. Nucleic acids Res. 2017, 45, D362-D368. [CrossRef] [PubMed] 
32. Shannon, P.; Markiel, A.; Ozier, O.; Baliga, N.S.; Wang, J.T.; Ramage, D.; Amin, N.; Schwikowski, B.; Ideker, T. Cytoscape: A software environment for integrated models of biomolecular interaction networks. Genome Res. 2003, 13, 2498. [CrossRef] [PubMed]

33. Fletcher, J.C.; Brand, U.; Running, M.P.; Simon, R.; Meyerowitz, E.M. Signaling of cell fate decisions by CLAVATA3 in Arabidopsis shoot meristems. Science 1999, 283, 1911-1914. [CrossRef] [PubMed]

34. Matsubayashi, Y. posttranslationally modified small-peptide signals in plants. Annu. Rev. Plant Biol. 2014, 65, 385-413. [CrossRef] [PubMed]

35. Lin, T.F.; Saiga, S.; Abe, M.; Laux, T. OBE3 and WUS interaction in shoot meristem stem cell regulation. PLoS ONE 2016, 11, e0155657. [CrossRef] [PubMed]

36. Della, R.F.; Fattorini, L.; D'Angeli, S.; Veloccia, A.; Del, D.S.; Cai, G.; Falasca, G.; Altamura, M.M. Arabidopsis SHR and SCR transcription factors and AUX1 auxin influx carrier control the switch between adventitious rooting and xylogenesis in planta and in vitro cultured thin cell layers. Ann. Bot. 2015, 115, 617-628. [CrossRef] [PubMed]

37. Wu, X.; Chory, J.; Weigel, D. Combinations of WOX activities regulate tissue proliferation during Arabidopsis embryonic development. Dev. Biol. 2007, 309, 306-316. [CrossRef] [PubMed]

38. Cheng, S.; Huang, Y.; Zhu, N.; Zhao, Y. The rice WUSCHEL-related homeobox genes are involved in reproductive organ development, hormone signaling and abiotic stress response. Gene 2014, 549, $266-274$. [CrossRef] [PubMed]

39. Liu, J.; Sheng, L.; Xu, Y.; Li, J.; Yang, Z.; Huang, H.; Xu, L. WOX11 and 12 are involved in the first-step cell fate transition during de novo root organogenesis in Arabidopsis. Plant Cell 2014, 26, 1081-1093. [CrossRef] [PubMed]

40. Dolzblasz, A.; Nardmann, J.; Clerici, E.; Causier, B.; van der Graaff, E.; Chen, J.; Davies, B.; Werr, W.; Laux, T. Stem cell regulation by Arabidopsis WOX genes. Mol. Plant 2016, 9, 1028-1039. [CrossRef] [PubMed]

41. Zhang, F.; Wang, Y.; Li, G.; Tang, Y.; Kramer, E.M.; Tadege, M. STENOFOLIA recruits TOPLESS to repress ASYMMETRIC LEAVES2 at the leaf margin and promote leaf blade outgrowth in Medicago truncatula. Plant Cell 2014, 26, 650-664. [CrossRef] [PubMed]

42. Zhao, Y.; Hu, Y.; Dai, M.; Huang, L.; Zhou, D.X. The WUSCHEL-related homeobox gene WOX11 is required to activate shoot-borne crown root development in rice. Plant Cell 2009, 21, 736-748. [CrossRef] [PubMed]

43. Berardini, T.Z.; Reiser, L.; Li, D.; Mezheritsky, Y.; Muller, R.; Strait, E.; Huala, E. The Arabidopsis information resource: Making and mining the "gold standard" annotated reference Plant Genome. Genesis 2015, 53, 474. [CrossRef] [PubMed]

44. Thompson, J.D.; Gibson, T.J.; Plewniak, F.; Jeanmougin, F.; Higgins, D.G. The CLUSTAL_X windows interface: Flexible strategies for multiple sequence alignment aided by quality analysis tools. Nucleic acids Res. 1997, 25, 4876-4882. [CrossRef] [PubMed]

45. Artimo, P.; Jonnalagedda, M.; Arnold, K.; Baratin, D.; Csardi, G.; de Castro, E.; Duvaud, S.; Flegel, V.; Fortier, A.; Gasteiger, E.; et al. ExPASy: SIB bioinformatics resource portal. Nucleic Acids Res. 2012, 40, W597-W603. [CrossRef] [PubMed]

46. Chou, K.C.; Shen, H.B. Cell-PLoc: A package of Web servers for predicting subcellular localization of proteins in various organisms. Nat. Protoc. 2007, 3, 153-162. [CrossRef] [PubMed]

47. Bailey, T.L.; Boden, M.; Buske, F.A.; Frith, M.; Grant, C.E.; Clementi, L.; Ren, J.; Li, W.W.; Noble, W.S. MEME SUITE: Tools for motif discovery and searching. Nucleic Acids Res. 2009, 37, W202-W208. [CrossRef] [PubMed]

48. Zhang, J.; Zhang, S.; Li, H.; Du, H.; Huang, H.; Li, Y.; Hu, Y.; Liu, H.; Liu, Y.; Yu, G. Identification of transcription factors ZmMYB111 and ZmMYB148 involved in phenylpropanoid metabolism. Front. Plant Sci. 2016, 7, 148. [CrossRef] [PubMed]

49. Livak, K.J.; Schmittgen, T.D. Analysis of relative gene expression data using real-time quantitative PCR and the $2^{-\Delta \Delta C t}$ method. Methods 2001, 25, 402-408. [CrossRef] [PubMed]

(c) 2018 by the authors. Licensee MDPI, Basel, Switzerland. This article is an open access article distributed under the terms and conditions of the Creative Commons Attribution (CC BY) license (http://creativecommons.org/licenses/by/4.0/). 